GLASNIK MATEMATIČKI

Vol. 39(59)(2004), $139-144$

\title{
A GENERALIZATION OF THE 0-NUMERICAL RANGE
}

\author{
RAJNA RAJIĆ \\ University of Zagreb, Croatia
}

\begin{abstract}
Let $H$ be a complex Hilbert space. Given a bounded linear operator $A$ on $H$, we describe the set $R^{n}(A)=\left\{V^{*} A W: V, W\right.$ : $\left.\mathbf{C}^{n} \rightarrow H, V^{*} V=W^{*} W=I_{n}, V^{*} W=0\right\}$. It is shown that the closed matricial convex hull of $R^{n}(A)$ is a closed ball of radius $\min \{\|A-\lambda I\|$ : $\lambda \in \mathbf{C}\}$ centered at the origin.
\end{abstract}

\section{INTRODUCTION}

Throughout this paper $H$ will denote a complex Hilbert space with an inner product $(\cdot, \cdot)$. By $B(H)$ we denote the algebra of all bounded linear operators on $H$.

In [15] E. L. Stolov showed that the 0-numerical range of a linear operator $A$ acting on a finite dimensional Hilbert space $H$ (i.e., the set $W_{0}(A)=$ $\{(A x, y): x, y \in H,(x, x)=(y, y)=1,(x, y)=0\})$ is a circular disc with center at the origin and with radius $\min \{\|A-\lambda I\|: \lambda \in \mathbf{C}\}$. The infinite dimensional analogue of this theorem was given in [8, Proposition 2.11].

In this paper we will consider the matricial generalization of the 0 numerical range of $A \in B(H)$. More precisely, our aim is to provide for $R^{n}(A)=\left\{V^{*} A W: V, W: \mathbf{C}^{n} \rightarrow H, V^{*} V=W^{*} W=I_{n}, V^{*} W=0\right\}$ a theorem analogous to the theorem of E. L. Stolov.

One obvious consequence of Stolov's theorem is that $\sup \left\{|\lambda|: \lambda \in W_{0}(A)\right\}$ is equal to $\min \{\|A-\lambda I\|: \lambda \in \mathbf{C}\}$. (For hermitian $A \in B(H)$ this result was first obtained by Mirsky ([11]).) As it will be seen, the same assertion is valid for the set $R^{n}(A)$.

2000 Mathematics Subject Classification. 47A12.

Key words and phrases. q-numerical range of an operator. 


\section{MAIN RESUlT}

Definition 2.1. For an operator $T \in B(H)$ we define the set

$$
R^{n}(A)=\left\{V^{*} A W: V, W: \mathbf{C}^{n} \rightarrow H, V^{*} V=W^{*} W=I_{n}, V^{*} W=0\right\} .
$$

REMARK 2.2. Observe that the operators $V$ and $W$ from the above definition are isometries from $\mathbf{C}^{n}$ to $H$ with orthogonal ranges. Therefore, to avoid the trivial case $R^{n}(A)=\emptyset$, we shall assume that the dimension of $H$ is greater than or equal to $2 n$.

REMARK 2.3. Note that $x$ and $y$ are orthogonal unit vectors of $H$ if and only if $V, W: \mathbf{C} \rightarrow H$, where $V(1)=y$ and $W(1)=x$, are isometries with orthogonal ranges. Then (identifying $B(\mathbf{C})$ with $\mathbf{C}$ ) we have $V^{*} A W=$ $(A x, y)$. So, in the case $n=1$ the set $R^{1}(A)$ coincides to the 0 -numerical range of an operator $A$. (For the definition and more details see $[8,10,15,16]$ ).

REMARK 2.4. Similar concept to the set $R^{n}(A)$ is the spatial matricial range of $A \in B(H)$ defined by $V^{n}(A)=\left\{V^{*} A V: V: \mathbf{C}^{n} \rightarrow H, V^{*} V=\right.$ $\left.I_{n}\right\}$. When $n=1$ this set reduces to the classical numerical range of $A$, i.e., $W(A)=\{(A x, x): x \in H,\|x\|=1\}$. However, the set $V^{n}(A)$ lacks an important property of $W(A)$ : it need not be convex if $n>1$ ([4, p. 142]). The closure of $W(A)$, known as the numerical range of $A$, is the set of all $\phi(A)$, where $\phi$ ranges over all norm-one positive linear functionals on $B(H)$. Using completely positive maps, W. B. Arveson ([1]) generalized the concept of numerical range in defining matricial range. J. Bunce and N. Salinas proved in [5, Theorem 3.5] that the matricial convex hull of $V^{n}(A)$ has the matricial range of $A$ as its closure. Basic references for the numerical and matricial ranges are $[1,3,4,5,6,7,13,14]$.

One other familiar concept is the set $\left\{V^{*} A W: V, W: \mathbf{C}^{n} \rightarrow H, V^{*} V=\right.$ $\left.W^{*} W=I_{n}\right\}$ where $H$ is a finite dimensional space which dimension is greater than or equal to $n$. In [9] the authors examine the conditions on $A$ under which this set is convex or starshaped.

REMARK 2.5. If $H$ is a finite dimensional space then $R^{n}(A)$ is a compact set. Indeed, let us take an arbitrary sequence $\left(V_{i}^{*} A W_{i}\right)_{i}$ in $R^{n}(A)$. Since $\left(V_{i}\right)$ and $\left(W_{i}\right)$ are the bounded sequences of isometries in the finite dimensional space $B\left(\mathbf{C}^{n}, H\right)$ of all linear operators from $\mathbf{C}^{n}$ to $H$ such that $V_{i}^{*} W_{i}=0$ they have the subsequences which converge to some isometries in $B\left(\mathbf{C}^{n}, H\right)$ with orthogonal ranges. Therefore, $\left(V_{i}^{*} A W_{i}\right)_{i}$ must also have a subsequence that converges in $R^{n}(A)$. Hence, $R^{n}(A)$ is compact.

Before stating our results we introduce some notation. 
The matricial convex hull of a subset $S$ of $B\left(\mathbf{C}^{n}\right)$, denoted by $\operatorname{mconv}(S)$, is the set of all finite sums of the form $\sum_{i} T_{i}^{*} A_{i} T_{i}$, where $A_{i} \in S$ and where the operators $T_{i} \in B\left(\mathbf{C}^{n}\right)$ are such that $\sum_{i} T_{i}^{*} T_{i}=I_{n}$.

We denote by $S^{-}$the topological closure of a set $S$.

The result which follows resembles those obtained by E. L. Stolov ([15]) and by C. K. Li, P. P. Mehta and L. Rodman ([8, Proposition 2.11]).

Theorem 2.6. Let $A \in B(H)$. Then

$$
\operatorname{mconv}\left(R^{n}(A)^{-}\right)=\left(\operatorname{mconv}\left(R^{n}(A)\right)\right)^{-}=\left\{L \in B\left(\mathbf{C}^{n}\right):\|L\| \leq r\right\},
$$

where $r=\min \{\|A-\lambda I\|: \lambda \in \mathbf{C}\}$. Particularly, if $H$ is finite dimensional then

$$
\operatorname{mconv}\left(R^{n}(A)\right)=\left\{L \in B\left(\mathbf{C}^{n}\right):\|L\| \leq r\right\} .
$$

Proof. The first equality follows by [6, Corollary 2.5] since $R^{n}(A)$ is a bounded subset of $B\left(\mathbf{C}^{n}\right)$ and $\mathbf{C}^{n}$ is finite dimensional.

Take any $V^{*} A W \in R^{n}(A)$. Since $V^{*} W=0$, for every $\lambda \in \mathbf{C}$ we have

$$
\left\|V^{*} A W\right\|=\left\|V^{*}(A-\lambda I) W\right\| \leq\left\|V^{*}\right\|\|A-\lambda I\|\|W\|=\|A-\lambda I\| .
$$

Hence, $\left\|V^{*} A W\right\| \leq \min \{\|A-\lambda I\|: \lambda \in \mathbf{C}\}=r$. We conclude that $R^{n}(A) \subseteq$ $\left\{L \in B\left(\mathbf{C}^{n}\right):\|L\| \leq r\right\}$. Since $\left\{L \in B\left(\mathbf{C}^{n}\right):\|L\| \leq r\right\}$ is a compact matricially convex set, it follows that $\left(\operatorname{mconv}\left(R^{n}(A)\right)\right)^{-} \subseteq\left\{L \in B\left(\mathbf{C}^{n}\right)\right.$ : $\|L\| \leq r\}$.

Recall that the unit ball in $B\left(\mathbf{C}^{n}\right)$ is the closed convex hull of the set of all unitary operators of $B\left(\mathbf{C}^{n}\right)$ ([12, Proposition 1.1.12]). Therefore, for the opposite inclusion it is enough to show that $\left(\operatorname{mconv}\left(R^{n}(A)\right)\right)^{-}$contains every normal operator in $B\left(\mathbf{C}^{n}\right)$ whose norm is less than or equal to $r$. Hence, let $L$ be a normal operator in $B\left(\mathbf{C}^{n}\right)$ with $\|L\| \leq r$. Denote by $\left\{e_{1}, \ldots, e_{n}\right\}$ an orthonormal basis of $\mathbf{C}^{n}$ consisting of eigenvectors of $L$. Let $\lambda_{i}$ be the eigenvalue of $L$ corresponding to $e_{i}$ and let $P_{i} \in B\left(\mathbf{C}^{n}\right)$ be the orthogonal projection on the subspace spanned by $e_{i}, i=1, \ldots, n$. Clearly, $L=\sum_{i=1}^{n} \lambda_{i} P_{i}$ and $\sum_{i=1}^{n} P_{i}=I_{n}$. Given $0<\varepsilon<1$ we get $\left|\lambda_{i}-\varepsilon \lambda_{i}\right|=(1-\varepsilon)\left|\lambda_{i}\right| \leq(1-\varepsilon)\|L\| \leq$ $(1-\varepsilon) r<r$, so by [15] (i.e. [8, Proposition 2.11]) there exist two orthogonal unit vectors $x_{i}, y_{i} \in H$ such that

$$
\lambda_{i}-\varepsilon \lambda_{i}=\left(A x_{i}, y_{i}\right)
$$

for $i=1, \ldots, n$. Now, for $x_{i}, y_{i} \in H$ and a unit vector $e_{i}$ one can find two isometries $V_{i}, W_{i}: \mathbf{C}^{n} \rightarrow H$ with orthogonal ranges such that $V_{i} e_{i}=y_{i}$ and 
$W_{i} e_{i}=x_{i}, i=1, \ldots, n$. From this we have $\left(V_{i}^{*} A W_{i} e_{i}, e_{i}\right)=\left(A x_{i}, y_{i}\right)$, so $P_{i} V_{i}^{*} A W_{i} P_{i}=\left(A x_{i}, y_{i}\right) P_{i}$. Therefore,

$$
L=\sum_{i=1}^{n} \lambda_{i} P_{i}=\sum_{i=1}^{n}\left(A x_{i}, y_{i}\right) P_{i}+\sum_{i=1}^{n} \varepsilon \lambda_{i} P_{i}=\sum_{i=1}^{n} P_{i} V_{i}^{*} A W_{i} P_{i}+\varepsilon L,
$$

so we obtain

$$
\left\|L-\sum_{i=1}^{n} P_{i} V_{i}^{*} A W_{i} P_{i}\right\|=\|\varepsilon L\| \leq \varepsilon r .
$$

Hence, the arbitrariness of $0<\varepsilon<1$ implies $L \in\left(\operatorname{mconv}\left(R^{n}(A)\right)\right)^{-}$.

The second assertion follows from the first one and Remark 2.5.

Given a bounded linear operator $A$ defined on a complex Hilbert space $H$, Mirsky's constant of $A$ ([11]), i.e.,

$$
\sup \{|(A x, y)|: x, y \in H,(x, x)=(y, y)=1,(x, y)=0\}
$$

is equal to $\min \{\|A-\lambda I\|: \lambda \in \mathbf{C}\}$, which is an obvious consequence of the result of [15] (see also [8, Proposition 2.11]). In what follows we shall see that an analogous assertion holds for the set $R^{n}(A)$.

Theorem 2.7. Let $A \in B(H)$. Then

$$
\begin{gathered}
\sup \left\{\left\|V^{*} A W\right\|: V, W: \mathbf{C}^{n} \rightarrow H,\|V\|=\|W\|=1, V^{*} W=0\right\}= \\
=\sup \left\{\|L\|: L \in R^{n}(A)\right\}=\min \{\|A-\lambda I\|: \lambda \in \mathbf{C}\} .
\end{gathered}
$$

Proof. Let us denote

$$
\begin{gathered}
m_{1}(A)=\left\{\left\|V^{*} A W\right\|: V, W: \mathbf{C}^{n} \rightarrow H,\|V\|=\|W\|=1, V^{*} W=0\right\} \\
m_{2}(A)=\left\{\|L\|: L \in R^{n}(A)\right\} \\
r=\min \{\|A-\lambda I\|: \lambda \in \mathbf{C}\} .
\end{gathered}
$$

Since $V^{*} V=W^{*} W=I_{n}$ implies $\|V\|=\|W\|=1$, it follows that $m_{2}(A) \subseteq$ $m_{1}(A)$. Further, for $V, W: \mathbf{C}^{n} \rightarrow H,\|V\|=\|W\|=1, V^{*} W=0$ we have

$$
\left\|V^{*} A W\right\|=\left\|V^{*}(A-\lambda I) W\right\| \leq\left\|V^{*}\right\|\|A-\lambda I\|\|W\|=\|A-\lambda I\|
$$

for every $\lambda \in \mathbf{C}$, so $\left\|V^{*} A W\right\| \leq r$. Hence,

$$
\sup m_{2}(A) \leq \sup m_{1}(A) \leq r .
$$

If $r=0$ we are done. So assume that $r>0$. By [15] (i.e. [8, Proposition 2.11]) we conclude that for an arbitrary $0<\varepsilon \leq r$ there exist $x_{\varepsilon}, y_{\varepsilon} \in H$ such that $\left(x_{\varepsilon}, x_{\varepsilon}\right)=\left(y_{\varepsilon}, y_{\varepsilon}\right)=1,\left(x_{\varepsilon}, y_{\varepsilon}\right)=0,\left|\left(A x_{\varepsilon}, y_{\varepsilon}\right)\right|=r-\varepsilon$. Let $V_{\varepsilon}, W_{\varepsilon}: \mathbf{C}^{n} \rightarrow H$ be two isometries with mutually orthogonal ranges such that $V_{\varepsilon} e=y_{\varepsilon}$ and $W_{\varepsilon} e=x_{\varepsilon}$, where $e \in \mathbf{C}^{n}$ is an arbitrary unit vector. Then we obtain

$$
r-\varepsilon=\left|\left(A x_{\varepsilon}, y_{\varepsilon}\right)\right|=\left|\left(A W_{\varepsilon} e, V_{\varepsilon} e\right)\right|=\left|\left(V_{\varepsilon}^{*} A W_{\varepsilon} e, e\right)\right| \leq\left\|V_{\varepsilon}^{*} A W_{\varepsilon}\right\|,
$$

so $r=\sup m_{2}(A)$. To complete the proof, it remains to apply (2.1). 
REMARK 2.8. In the original manuscript a concept of a generalized numerical range equivalent to the one introduced in Definition 2.1 was described for operators on Hilbert $C^{*}$-modules. As it was pointed out by the referee this reduces to the case of Hilbert space operators (after representing a Hilbert $C^{*}$-module as a concrete space of operators). However, in our subsequent paper we shall present some results concerned with the generalized numerical ranges for operators on Hilbert $C^{*}$-modules that can be obtained by the methods based on the results of [2].

\section{ACKNOWLEDGEMENTS.}

The author would like to thank Professor Damir Bakić for his useful comments and helpful advices. Thanks are also due to the referee for his/her valuable suggestions.

\section{REFERENCES}

[1] W. B. Arveson, Subalgebras of $C^{*}$-algebras II, Acta Math. 128 (1972), 271-308.

[2] D. Bakić and B. Guljaš, Hilbert $C^{*}$-modules over $C^{*}$-algebras of compact operators, Acta Sci. Math. (Szeged) 68 (2002), 249-269.

[3] F. F. Bonsall and J. Duncan, Numerical ranges of operators on normed spaces and of elements of normed algebras, London Math. Soc. Lecture Note Series 2, Cambridge University Press, Cambridge, 1971.

[4] F. F. Bonsall and J. Duncan, Numerical ranges II, London Math. Soc. Lecture Note Series 10, Cambridge University Press, Cambridge, 1973.

[5] J. Bunce and N. Salinas, Completely positive maps on $C^{*}$-algebras and the left matricial spectra of an operator, Duke Math. J. 43 (1976), 747-774.

[6] D. R. Farenick, $C^{*}$-convexity and matricial ranges, Can. J. Math. Vol. 44(2) (1992), 280-297.

[7] R. A. Horn and C. R. Johnson, Topics in Matrix Analysis, Cambridge University Press, Cambridge, 1991.

[8] C. K. Li, P. P. Mehta and L. Rodman, A generalized numerical range: the range of a constrained sesquilinear form, Linear and Multilinear Algebra 37 (1994), 25-49.

[9] C. K. Li and N. K. Tsing, On the kth matrix numerical range, Linear and Multilinear Algebra 28 (1991), 229-239.

[10] M. Marcus and P. Andresen, Constrained extrema of bilinear functionals, Monatsh. Math. 84 (1977), 219-235.

[11] L. Mirsky, Inequalities for normal and hermitian matrices, Duke Math. J. 24 (1957), 591-599.

[12] G. K. Pedersen, $C^{*}$-algebras and their automorphisms groups, Academic Press, New York, 1979.

[13] R. R. Smith and J. D. Ward, Matrix ranges for Hilbert space operators, Amer. J. Math. 102 (1980), 1031-1081.

[14] J. G. Stampfli and J. P. Williams, Growth conditions and the numerical range in a Banach algebra, Tohôku Math. J. 20 (1968), 417-424.

[15] E. L. Stolov, On Hausdorff set of matrices, Izv. Vyssh. Ucebn. Zaved. Mat. 10 (1979), 98-100.

[16] N. K. Tsing, The constrained bilinear form and the C-numerical range, Linear Algebra Appl. 56 (1984), 195-206. 
Faculty of Mining, Geology and Petroleum Engineering

University of Zagreb

Pierottijeva 6, 10000 Zagreb

Croatia

E-mail: rajna.rajic@zg.hinet.hr

Received: 11.12.2002

Revised: 05.03.2003 